\title{
Protective Effect of Guggulipid in High Fat Diet and Middle Cerebral Artery Occlusion (MCAO) Induced Ischemic Cerebral Injury in Rats
}

Authors

Affiliations

\author{
M. A. Ahmad' ${ }^{1}$, A. K. Najmi' ${ }^{1}$ M. Mujeeb ${ }^{2}$, M. Akhtar ${ }^{1}$
}

${ }^{1}$ Department of Pharmacology, Faculty of Pharmacy, Jamia Hamdard, New Delhi India

${ }^{2}$ Department of Pharmacognosy and Phytochemistry, Faculty of Pharmacy, Jamia Hamdard, New Delhi India

\author{
Key words \\ - guggulipid \\ - aspirin \\ - HFD \\ - MCAO \\ - cerebral ischemia
}

received 01.03 .2016

accepted 22.04.2016

\section{Bibliography}

DOI http://dx.doi.org/ 10.1055/s-0042-107787

Published online:

May 25, 2016

Drug Res 2016;

66: 407-414

(c) Georg Thieme Verlag KG

Stuttgart · New York

ISSN 2194-9379

\section{Correspondence}

\section{Dr. Mohd. Akhtar}

Department of Pharmacology

Faculty of Pharmacy

Jamia Hamdard (Hamdard

University)

M. B Road

Hamdard Nagar

New Delhi 10062

India

Tel.: + 91/011/26059688

Fax: + 91/011/26059666

Mobile: +91/9811777 883

makhtar_ph@jamiahamdard.

ac.in

fiatakhtar@hotmail.com

\begin{abstract}
$\nabla$

Aim: The role of guggulipid was evaluated in high fat diet and middle cerebral artery occlusion (MCAO) induced ischemic cerebral dysfunctions in rats of either sex.

Materials and Methods: Ethyl acetate extract of guggul known as guggulipid was prepared and administered to rats. Animals were divided into 9 groups, consisting 6 rats, each receiving different treatments per orally for 8 weeks. Control group rats received normal control diet while rest of the other groups animals were fed high fat diet (HFD) for 8 weeks. Cerebral ischemia was induced for $2 \mathrm{~h}$ followed by reperfusion for $22 \mathrm{~h}$. Locomotor activity and grip strength tests were performed immediately after $24 \mathrm{~h}$ of reperfusion followed by biochemical estimations and histopathology.
\end{abstract}

\section{Introduction}

\section{$\nabla$}

Cerebral ischemia (stroke) is responsible for severe morbidity and mortality worldwide [1]. In India itself $2 \%$ of total deaths occurred due to the stroke [2]. According to American Stroke Association, large-artery atherosclerosis $(20.9 \%)$ was the most common cause of stroke in middle-aged patients and left ventricular thrombus or thromboembolism was another important risk factor for ischemic stroke [3].

Obesity is an independent risk factor for acute ischemic stroke (AIS) including other risk factors such as hypertension, diabetes and hyperlipidemia [3]. Experimental studies showed that either high fat diet (HFD) or genetically induced obesity was accompanied with increased cerebrovascular change which produced hypertension and increased infarct size in either transient or permanent focal ischemic model $[4,5]$.

Clinical studies also suggested that obesity is an independent predictor of unfavorable functional
Results: Locomotor activity and grip strength were significantly decreased in HFD and HFD fed MCAO groups and improved significantly in pretreatment groups. Cerebral infarction, thiobarbituric acid reactive substances (TBARs), nitric oxide and tumor necrosis factor alfa (TNF $\alpha$ ) levels were increased, pretreatment of guggulipid alone and with aspirin significantly reduced these markers. Reduced glutathione (GSH), superoxide dismutase (SOD) and catalase, levels were decreased but all drug pretreated groups showed significant improvement in those markers.

Conclusion: Guggulipid demonstrated neuroprotection owing to its hypolipidemic, antioxidant, anti-inflammatory and anti-thrombotic activities but further research is warranted to confirm its role in cerebral ischemia.

outcome and mortality in AIS patients treated with tissue plasminogen activator (t-PA), the only therapeutic option available $[5,6]$. Diet induced obesity was also reported caused by ischemic stroke [7]. One of the study reported that the high fat diet causes significantly greater loss of cortical tissue post cortical contusion injury (CCI) than in the standard diet group [8]. Rt-PA $(0.9 \mathrm{mg} / \mathrm{kg}, \mathrm{iv})$, is the only drug approved by FDA, it has potential side effects and is the drug reserve for emergency treatment within $3 \mathrm{~h}$ of onset of ischemic stroke [9]. This indicates the limited resources available for improving ischemic stroke mortality and morbidity.

Drug therapy of stroke provides prophylactic protection. It has been seen that in animal models of stroke with many drugs pre-treatment, yields better outcome than post onset treatment [10].

According to Ministry of Health Clinical Practice Guidelines, Anti-platelet therapy is recommended in all patients with ischemic stroke to 
reduce deep venous thrombosis and pulmonary embolism. Patients with ischemic stroke or transient ischemic attack are treated with statins group of hypolipidemic drugs to reduce the risk of vascular outcomes [11].

Aspirin is the most commonly used drug in patients with coronary heart diseases. It is used as first line treatment for transient ischemic attacks. According to Clinical Practice Guidelines, aspirin is prescribed immediately for patients who have sustained ischemic stroke $[1,12,13]$. Therefore, in this study, aspirin and atorvastatin were taken as reference standard drugs.

The gum resin from the tree Commiphora whighitii has been used for thousands of years in the treatment of arthritis, inflammation, obesity, cardiac protection, anti-ulcer, anti-epileptic and disorders of lipid metabolism. Commiphora whighitii is a member of the Burseraceae family and is found in arid areas of India, Bangladesh, and Pakistan. Guggulipid is an ethyl acetate extract of resin from the tree Commiphora whighitii [14].

Clinical studies also support that guggulipid showed hypolipidemic effect (Farnesoid $\mathrm{X}$ receptor antagonist), anti-oxidant effect, anti-inflammatory effect due to inhibition of activation of NF-kappa B (which is responsible for ischemia induced neuronal injury) [15] and cardioprotective effect in ischemic patients [16].

Preclinical studies reported that guggulipid inhibited the platelet aggregation [17], had anti-inflammatory effect (via inhibition of TNF $\alpha$ expression, which was elevated in stroke) [18], delayed progression of atherosclerosis, impaired plasminogen activator inhibitor-1 as well as vascular cell adhesion molecule- 1 protein [19], and anti-hypercholesterolemic [14] (as Farnesoid X receptor antagonist).

So, the present study was carried out to assess the effects of guggulipid in high fat diet and middle cerebral artery occlusion (MCAO) induced ischemic cerebral dysfunctions in rats to explore its potential to be used in cerebral ischemia.

\section{Materials and Methods}

Experimental animals

The study was approved by the Institutional Animal Ethics Committee (IAEC) of Hamdard University, New Delhi (Protocol No. 788/2012). Albino Wistar rats of either sex with body weight 250-300g were procured from Central Animal House Facility, Hamdard University, New Delhi and accommodated under standard laboratory conditions at a temperature $\left(20-25^{\circ} \mathrm{C}\right)$, illumination (12-h light and 12-h dark: day: night cycle, manually controlled) and had provided diet (normal pellet diet for control group and high fat diet for other groups) and water.

\section{Drugs procurements}

Guggulipid is an ethyl acetate extract of the resin of plant Commiphora whighitii or guggul was prepared. Guggul was purchased from Khari Bawli market, Old Delhi. The guggul was identified by Dr. H. B. Singh at National Institute of Science Communication and Information Resources (NISCAIR), New Delhi. The collected drug sample voucher specimen (Ref.No. NISCAIR/ RHMD/Consult/2011-12/1081/281) was obtained and deposited at NISCAIR, New Delhi, India. Guggulipid extract was prepared and standardized with high-performance thin-layer chromatography (HPTLC) analysis in Department of Pharmacognosy and Phytochemistry lab Jamia Hamdard. The composition of high fat diet ( $\mathrm{g} / \mathrm{kg}$ body weight) include: Casein-342g; Cystine-3g;
Starch-172 g; Sucrose-172 g; Cellulose-50g; G.N. oil-25g; Tallow-190 g; Mineral oil-37g; Vitamin Mix-10g. High fat diet was procured from National Center for Laboratory Animal Sciences (NCLAS), National Institute of Nutrition, Hyderabad, India.

\section{Experimental schedule}

After acclimatization, all the animals were randomly allocated into 9 groups of 6 animals each and treatment schedule was as followed: Group I received normal diet with physiological saline served as control. Group II received high fat diet for 8 weeks [8] with physiological saline, as high fat diet per se group. Groups III, in this group rats were subjected to cerebral ischemia by middle cerebral artery occlusion after receiving high fat diet for 8 weeks with physiological saline named as high fat diet + MCAO group. Groups IV, in this group rat were induced ischemia by middle cerebral artery occlusion after receiving high fat diet for 8 weeks with guggulipid dose $1(50 \mathrm{mg} / \mathrm{kg})$ named as HFD + GL1 + MCAO group. Groups V, in this group rat were induced ischemia by middle cerebral artery occlusion after receiving high fat diet for 8 weeks with Guggulipid dose $2(100 \mathrm{mg} / \mathrm{kg})$ named as HFD + GL2 + MCAO group. Group VI, in this group rat were induced ischemia by middle cerebral artery occlusion after receiving high fat diet for 8 weeks with Atorvastatin $(20 \mathrm{mg} / \mathrm{kg})$ named as HFD + atorvastatin + MCAO group.

Groups VII, in this group rat were induced ischemia by middle cerebral artery occlusion after receiving high fat diet for 8 weeks with Aspirin (100 mg/kg) as HFD + ASP + MCAO group.

Groups VIII, in this group rat were induced ischemia by middle cerebral artery occlusion after receiving high fat diet for 8 weeks with guggulipid dose 1 and Aspirin $(100 \mathrm{mg} / \mathrm{kg})$ as HFD+GL1+ ASP+MCAO group. Groups IX, in this group rat were induced ischemia by middle cerebral artery occlusion after receiving high fat diet for 8 weeks with Guggulipid dose 2 and Aspirin (100 mg/kg) as HFD + GL 2 + ASP + MCAO group.

After $3 \mathrm{~h}$ of last dose of all drugs administration, cerebral ischemia was induced for $2 \mathrm{~h}$ followed by reperfusion for $22 \mathrm{~h}$. The rats were observed for behavioral parameters and then immediately sacrificed for infarct areas and estimation of biochemical parameters in brain.

\section{Induction of cerebral ischemia}

Chloral hydrate $(400 \mathrm{mg} / \mathrm{kg}$, i.p) was administered to anesthetize the rats. A mid-line incision was done in the neck region and the right common carotid artery, external carotid artery and internal carotid artery were exposed under an operative magnifying glass. A 4.0 monofilament nylon thread (40-3033pk10, Doccol Corporation, Redlands, CA) was inserted from the external carotid artery into the lumen of the internal carotid artery until resistance was felt, which confirms the occlusion of the origin of the middle cerebral artery [20]. The nylon filament was allowed to remain in place for $2 \mathrm{~h}$. The filament was retracted after $2 \mathrm{~h}$ to allow the reperfusion of ischemic region. Adequate precautions were taken to prevent the infection. After $24 \mathrm{~h}$, the animals were studied for neurobehavioral activities like locomotor activity and grip strength. Immediately after behavioral tests, the animals were sacrificed, their brain was removed, infarct size was measured, homogenate was prepared from the brain slices and biochemical estimations were carried out.

\section{Open field locomotor activity}

It was observed in open field arenas consisting of an acrylic box $\left(40.6 \times 40.6 \times 40.6 \mathrm{~cm}^{3}\right)$ accommodated with 2 photo beam 
frames (16 beams/dimensions; $2.5 \mathrm{~cm}$ between beams; Coulbourn Instruments, Allentown, PA). The horizontal locomotor activity was recorded by the lower frame $(2.5 \mathrm{~cm}$ above the arena floor) while the upper frame $(15 \mathrm{~cm}$ above the floor) records rearing. The open field chamber was joined to a computer running software (TruScan 2.0 version, Coulbourn Instruments, Allentown, PA) that recorded beam breaks $(100 \mathrm{~ms}$ sampling rate). Rats were kept for half an hour in home cage for habituation. Then they were placed in an open field chamber for half an hour prior to observe the locomotor activity [21,22]. Locomotion activity was recorded for 20 min during which horizontal locomotion activity was recorded of each rat.

\section{Grip strength}

Grip strength meter was used for recording the grip strength of the animal [23]. The animal's front paws were placed on the grid of grip strength meter and were moved down until its front paws grasping the grid were released. The force achieved by the animal was then displayed on the screen and was recorded as $\mathrm{Kg}$ unit.

\section{Biochemical estimations}

\section{Lipid peroxidation estimation}

Lipid peroxidation was estimated by production of thiobarbituric acid reactive substance (TBARs) as described by Ohkawa [24]. According to that, $0.1 \mathrm{ml}$ of homogenate, $1 \mathrm{ml}$ of trichloroacetic acid $(10 \% \mathrm{w} / \mathrm{v})$ and $1 \mathrm{ml}$ of thiobarbituric acid $(0.67 \% \mathrm{w} / \mathrm{v})$ were added to test tubes. All test tubes were covered with aluminum foil and placed in boiling water bath for $20 \mathrm{~min}$. Then, all test tubes were placed in crushed ice bath and centrifuged at $3000 \times \mathrm{g}$ for $10 \mathrm{~min}$. The absorbance of all the supernatant was measured at $540 \mathrm{~nm}$.

\section{Glutathione estimation}

Glutathione (GSH) was estimated by the method of Ellman [25]. The equal amount of brain homogenate $(\mathrm{w} / \mathrm{v})$ and $10 \%$ trichloroacetic acid were mixed and centrifuged. A $2 \mathrm{ml}$ of phosphate buffer (pH 7.4), $0.5 \mathrm{ml}$ 5,5-dithiobisnitro benzoic acid (DTNB) and $0.4 \mathrm{ml}$ of double-distilled water were added to $0.01 \mathrm{ml}$ of supernatant. Then, the mixture was vortexed and their absorbance was recorded at $412 \mathrm{~nm}$ within $15 \mathrm{~min}$.

\section{Superoxide dismutase estimation}

Superoxide dismutase activity was measured by the method of Beauchamp \& Fridovich [26]. The supernatant was assayed for SOD activity by following the inhibition of pyrogallol autoxidation. A $100 \mathrm{ml}$ of cytosolic supernatant was added to Tris- $\mathrm{HCl}$ buffer ( $\mathrm{pH} 8.5$ ). The final volume of $3 \mathrm{ml}$ was adjusted with the same buffer. At least $25 \mathrm{ml}$ of pyrogallol was added and changes in absorbance at $420 \mathrm{~nm}$ were recorded at $1 \mathrm{~min}$ interval for $3 \mathrm{~min}$. The increase in absorbance at $420 \mathrm{~nm}$ after the addition of pyrogallol was inhibited by the presence of SOD.

\section{Catalase estimation}

Catalase activity was measured by the method of Claiborne \& Greenworld [27]. A total of $0.1 \mathrm{ml}$ of supernatant was added to cuvette containing $1.9 \mathrm{ml}$ of $50 \mathrm{mM}$ phosphate buffer ( $\mathrm{pH} 7)$. The reaction was started by the addition of $1 \mathrm{ml}$ freshly prepared $30 \mathrm{mM} \mathrm{H} \mathrm{H}_{2}$. The rate of decomposition of $\mathrm{H}_{2} \mathrm{O}_{2}$ was measured spectrophotometrically at $240 \mathrm{~nm}$.

\section{Nitrite estimation}

The accumulation of nitrite in the brain supernatant, an indicator of the production of nitric oxide (NO), was determined by a colorimetric assay with Griess reagent (0.1\% N-(1-naphthyl) ethylene diamine dihydrochloride, $1 \%$ sulfanilamide and $2.5 \%$ phosphoric acid [28]. Equal volumes of Griess reagent and supernatant were mixed after that the mixture was incubated for $10 \mathrm{~min}$ at room temperature in the dark, and the absorbance at $540 \mathrm{~nm}$ was determined with Perkin Elmer lambda $20 \mathrm{spec}-$ trophotometer. The concentration of nitrite in the supernatant was determined from a sodium nitrite standard curve and expressed as percentage of control.

$\mathrm{TNF}_{\alpha}$ estimation by enzyme-linked immunosorbent assay (ELISA)

Rats were sacrificed after $24 \mathrm{~h}$ of middle cerebral occlusion and the brain homogenates were obtained. The contents of TNF $\alpha$ were measured in tissue homogenates using specific ELISA kits according to the manufactures instructions (Krishgen Biosystems).

\section{Measurement of cerebral infarct area}

After $24 \mathrm{~h}$ of middle cerebral artery occlusion, rats were sacrificed and their brains were removed. The brains were sectioned into 6 slices each with $2 \mathrm{~mm}$ thickness, immersed in $2 \%$ Triphenyl Tetrazolium Chloride (TTC) dye for $30 \mathrm{~min}$ at a $37^{\circ} \mathrm{C}$. The brain tissue was differentiated according to white-colored infarct area and red-purple non-infarct area. The slices were placed on a glass plate in a row and the images were captured. Image J Software (NIH, Bethesda, MD) was used for the measurement of infarction (white area) and the total area of the section (red-purple). The percentage of the area of infarction was calculated as [(white or yellow area/total area $) \times 100]$ [29].

\section{Statistical analysis}

All the values were expressed as the mean \pm SEM. All group means were compared by one-way analysis of variance (ANOVA) for statistical analysis followed by Dunnett's t-test. P-value $<0.05$ was considered significant. All the statistical analysis was carried out using graph pad in Stat 3 Software (The National Institute of Justice, Washington, DC).

\section{Results}

$\nabla$

Effect of pretreatment of guggulipid, aspirin, atorvastatin and their co-administration on locomotor activity in high fat diet fed middle cerebral artery occluded rats

Locomotor activity on video path analyzer was observed. Locomotor responses, such as move time, rest time, horizontal activity, mean velocity and total movement were observed. Mean time, horizontal activity, means velocity, total movements were significantly decreased in HFD and HFD + MCAO group as compared with control group, whereas rest time was significantly increased $(p<0.001)$. Locomotor responses such as mean time, horizontal activity, means velocity, total movements of pretreated guggulipid dose 1 and dose 2, aspirin, atorvastatin and guggulipid and aspirin combination group showing significant enhancement $(\mathrm{p}<0.01)$ as compared to HFD and HFD + MCAO groups, but rest time was significantly decreased ( $\bullet$ Table 1 ). 
Table 1 Effect of pretreatment of guggulipid, aspirin, atorvastatin and their co-administration on locomotor activity in high fat diet fed middle cerebral artery occluded rats.

\begin{tabular}{|c|c|c|c|c|c|}
\hline Groups $(\mathrm{N}=6)$ & Move time (s) & Rest time (s) & $\begin{array}{l}\text { Horizontal } \\
\text { activity (cm) }\end{array}$ & $\begin{array}{l}\text { Mean } \\
\text { velocity }(\mathrm{cm} / \mathrm{s})\end{array}$ & $\begin{array}{l}\text { Total } \\
\text { movement }\end{array}$ \\
\hline Control & $274 \pm 19.6$ & $181.02 \pm 14.54$ & $2554 \pm 56$ & $536 \pm 11.3$ & $625 \pm 27.4$ \\
\hline HFD per se & $157.4 \pm 12.26^{\# \#}$ & $315.6 \pm 10.86^{\# \#}$ & $1579 \pm 104^{\# \#}$ & $305 \pm 34.9^{\# \#}$ & $409 \pm 48.8^{\# \#}$ \\
\hline $\mathrm{HFD}+\mathrm{MCAO}$ & $86.6 \pm 11.7^{\# \# \#}$ & $426.2 \pm 19.69^{\# \# \#}$ & $915 \pm 85^{\# \# \#}$ & $187.4 \pm 30.4^{\# \# \#}$ & $277 \pm 42.16^{\# \# \#}$ \\
\hline $\mathrm{HFD}+\mathrm{GL}(50 \mathrm{mg} / \mathrm{kg})+\mathrm{MCAO}$ & $154 \pm 9.49 * *$ & $292.4 \pm 17.49^{* *}$ & $1552 \pm 107^{* *}$ & $323 \pm 15^{* *}$ & $430 \pm 29.6 * *$ \\
\hline $\mathrm{HFD}+\mathrm{GL}(100 \mathrm{mg} / \mathrm{kg})+\mathrm{MCAO}$ & $201 \pm 11.9^{* *}$ & $250.8 \pm 15.38^{* *}$ & $2015 \pm 113^{* *}$ & $403.2 \pm 12.7^{* *}$ & $488 \pm 24.39 * * *$ \\
\hline $\mathrm{HFD}+\mathrm{ASP}(100 \mathrm{mg} / \mathrm{kg})+\mathrm{MCAO}$ & $169 \pm 9.3 * *$ & $302.0 \pm 10.7^{* *}$ & $1668 \pm 106^{* *}$ & $315 \pm 21^{* *}$ & $418 \pm 22.34^{* *}$ \\
\hline $\mathrm{HFD}+\mathrm{ATORVA}(20 \mathrm{mg} / \mathrm{kg})+\mathrm{MCAO}$ & $175 \pm 12.1 * *$ & $295.0 \pm 14.7^{* *}$ & $1798 \pm 136^{* *}$ & $325 \pm 21^{* *}$ & $429 \pm 26.4^{* *}$ \\
\hline $\mathrm{HFD}+\mathrm{ASP}(100 \mathrm{mg} / \mathrm{kg})+\mathrm{GL}(50 \mathrm{mg} / \mathrm{kg})+\mathrm{MCAO}$ & $232.8 \pm 12.8^{* * *}$ & $245.0 \pm 9.1^{* * *}$ & $2131 \pm 87^{* * *}$ & $428 \pm 26 * * *$ & $519.8 \pm 24.7^{* * *}$ \\
\hline $\mathrm{HFD}+\mathrm{ASP}(100 \mathrm{mg} / \mathrm{kg})+\mathrm{GL}(100 \mathrm{mg} / \mathrm{kg})+\mathrm{MCAO}$ & $241.4 \pm 16.4^{* * *}$ & $193.0 \pm 11.4^{* * *}$ & $2243 \pm 56.3^{* * *}$ & $460 \pm 20 * * *$ & $552 \pm 38 * * *$ \\
\hline
\end{tabular}

All the values were expressed as the mean \pm SEM. All group means were compared by one-way analysis of variance (ANOVA) for statistical analysis followed by Dunnett's $t$-test. $\mathrm{N}=$ number of animals 6 , Analysis of Variance (ANOVA) was performed followed by Dunnett's't'-test. ${ }^{\#} \mathrm{p}<0.05,{ }^{\# \#} \mathrm{p}<0.01$ vs. Control; ${ }^{*} \mathrm{p}<0.05,{ }^{* *} \mathrm{p}<0.01$ vs. HFD +MCAO. HFD - high fat diet; MCAO - middle cerebral artery occlusion; GL - guggulipid; ASP - aspirin; ATORVA - atorvastatin

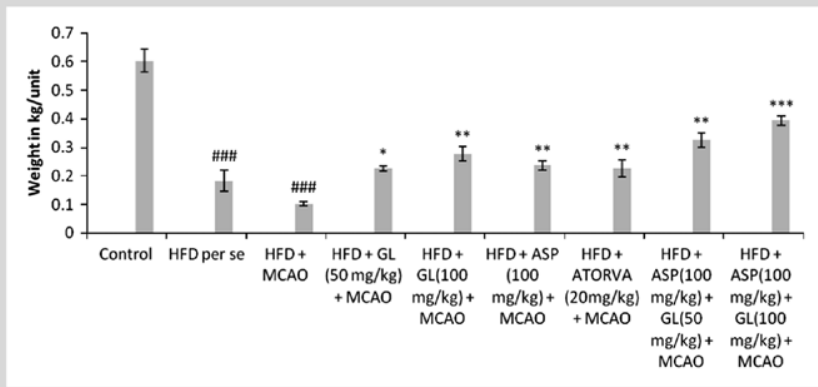

Fig. 1 Effect of pretreatment of guggulipid, aspirin, atorvastatin and their co-administration grip strength in high fat diet fed middle cerebral artery occluded rats. All the values were expressed as the mean \pm SEM. All group means were compared by one-way analysis of variance (ANOVA) for statistical analysis followed by Dunnett's t-test. $N=$ number of animals $6,{ }^{\# \# \#} \mathrm{p}<0.001$ vs. control group; ${ }^{*} \mathrm{p}<0.05,{ }^{* *} \mathrm{p}<0.01,{ }^{* * *} \mathrm{p}<0.001$ vs. HFD + MCAO. HFD - high fat diet, MCAO - middle cerebral artery occlusion, GL - guggulipid, ASP - aspirin, ATORVA - atorvastatin.

Effect of pretreatment of guggulipid, aspirin, atorvastatin and their co-administration on grip strength in high fat diet fed middle cerebral artery occluded rats

Grip strength was significantly decreased in HFD and HFD + MCAO groups $(\mathrm{p}<0.001)$ as compared with normal group. Grip strength was improved in pre-treated groups as compared with HFD and HFD+MCAO groups ( $\mathrm{p}<0.01 ; 0$ Fig. 1$)$.

Effect of pretreatment of guggulipid, aspirin, atorvastatin and their co-administration on TBARs in high fat diet fed middle cerebral artery occluded rats In this study TBARs levels were significantly increased in HFD and HFD + MCAO groups $(\mathrm{p}<0.001)$ as compared with normal group. TBARs levels were significantly decreased in pretreated groups of guggulipid dose 1 , guggulipid dose 2 , aspirin and atorvastatin than HFD and HFD + MCAO groups $(\mathrm{p}<0.01$; $\odot$ Table 2$)$.

Effect of pretreatment of guggulipid, aspirin, atorvastatin and their co-administration on GSH in high fat diet fed middle cerebral artery occluded rats The GSH levels were found to decrease significantly in the HFD and HFD + MCAO groups than normal group of rats $(p<0.01)$. The GSH levels were significantly elevated in pre-treated group of guggulipid, aspirin and atorvastatin followed by ischemia than those in HFD and HFD+MCAO groups $(\mathrm{p}<0.01)$. A combination of both guggulipid and aspirin showed a significant enhancement in the GSH level $(\mathrm{p}<0.01$; $\odot$ Table 2$)$.

Effect of pretreatment of guggulipid, aspirin, atorvastatin and their co-administration on SOD in high fat diet fed middle cerebral artery occluded rats In this study SOD levels were decreased significantly in the HFD and HFD + MCAO groups than normal group of rats $(p<0.01)$. The SOD levels were significantly elevated in pre-treated group of guggulipid, aspirin and atorvastatin than those in HFD and HFD + MCAO groups $(p<0.01)$. Combination of guggulipid and aspirin also showed a significant enhancement in the SOD level $(\mathrm{p}<0.01 ; \odot$ Table 2$)$.

Effect of pretreatment of guggulipid, aspirin, atorvastatin and their co-administration on catalase in high fat diet fed middle cerebral artery occluded rats Catalase levels were decreased significantly in the HFD and HFD + MCAO groups than normal group of rats $(p<0.01)$. After pretreatment with guggulipid, aspirin, atorvastatin and combination of guggulipid and aspirin it was observed that catalase levels were significantly increased $(\mathrm{p}<0.01 ; \odot$ Table 3$)$.

Effect of pretreatment of guggulipid, aspirin, atorvastatin and their co-administration on nitrite release in high fat diet fed middle cerebral artery occluded rats

In HFD and HFD+MCAO group nitrite levels were increased significantly than normal group of rats $(p<0.01)$. The nitrite levels were significantly decreased in pre-treated group of guggulipid, aspirin and atorvastatin than those in HFD and HFD+MCAO groups $(p<0.01)$. Combination of guggulipid and aspirin also showed a significant reduction in the nitrite level $(\mathrm{p}<0.01 ; \odot$ Table 3$)$.

Effect of pretreatment of guggulipid, aspirin, atorvastatin and their co-administration on $\mathrm{TNF}_{\alpha}$ in high fat diet fed middle cerebral artery occluded rats $\mathrm{TNF}_{\alpha}$ levels were significantly increased in HFD and HFD + MCAO group as compared with control group $(\mathrm{p}<0.01)$. The $\mathrm{TNF}_{\alpha}$ levels were significantly decreased in pre-treated group of guggulipid, aspirin and atorvastatin than those in HFD and HFD + MCAO groups $(p<0.01)$. Combination of guggulipid and aspirin also showed a significant reduction in the $\operatorname{TNF}_{\alpha}(\mathrm{p}<0.01 ; \bullet$ Fig. 2$)$. 
Table 2 Effect of pretreatment of guggulipid, aspirin, atorvastatin and their co-administration on SOD, TBARS and GSH in high fat diet fed middle cerebral artery occluded rats.

\begin{tabular}{|c|c|c|c|}
\hline Groups $(\mathrm{N}=6)$ & $\begin{array}{l}\text { TBARS ( } \mathrm{nmol} / \mathrm{mg} \text { of MDA } \\
\text { formed/g of protein) }\end{array}$ & GSH ( $\mu \mathrm{g} / \mathrm{g}$ of protein) & SOD (U/mg mol) \\
\hline Control & $2.516 \pm 0.25$ & $8.55 \pm 0.35$ & $171.8 \pm 15.9$ \\
\hline HFD per se & $3.91 \pm 0.34^{\#}$ & $4.3 \pm 0.44^{\#}$ & $116.41 \pm 24.7^{\#}$ \\
\hline $\mathrm{HFD}+\mathrm{MCAO}$ & $6.15 \pm 0.22^{\# \# \#}$ & $2.017 \pm 0.39 \#$ & 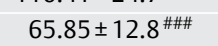 \\
\hline $\mathrm{HFD}+\mathrm{GL}(50 \mathrm{mg} / \mathrm{kg})+\mathrm{MCAO}$ & $4.003 \pm 0.09^{*}$ & $4.3 \pm 0.24^{*}$ & $119.59 \pm 11^{*}$ \\
\hline $\mathrm{HFD}+\mathrm{GL}(100 \mathrm{mg} / \mathrm{kg})+\mathrm{MCAO}$ & $3.28 \pm 0.43^{* *}$ & $5.17 \pm 0.52 * *$ & $135 \pm 9.3 * *$ \\
\hline $\mathrm{HFD}+\mathrm{ASP}(100 \mathrm{mg} / \mathrm{kg})+\mathrm{MCAO}$ & $3.28 \pm 0.43^{* *}$ & $4.45 \pm 0.22 * *$ & $118.5 \pm 20.3^{* *}$ \\
\hline $\mathrm{HFD}+\mathrm{ATORVA}(20 \mathrm{mg} / \mathrm{kg})+\mathrm{MCAO}$ & $4.16 \pm 0.12 *$ & $4.14 \pm 0.3^{*}$ & $106.99 \pm 15.08^{*}$ \\
\hline$H F D+A S P(100 \mathrm{mg} / \mathrm{kg})+G L(50 \mathrm{mg} / \mathrm{kg})+\mathrm{MCAO}$ & $2.93 \pm 0.14^{* *}$ & $6.04 \pm 0.27^{* *}$ & $147 \pm 8.4^{* *}$ \\
\hline $\mathrm{HFD}+\mathrm{ASP}(100 \mathrm{mg} / \mathrm{kg})+\mathrm{GL}(100 \mathrm{mg} / \mathrm{kg})+\mathrm{MCAO}$ & $2.61 \pm 0.08 * * *$ & $6.8 \pm 0.28 * *$ & $154.49 \pm 9.7 * * *$ \\
\hline
\end{tabular}

\begin{tabular}{|c|c|c|}
\hline Groups $(\mathrm{N}=6)$ & Nitrite release $(\mu \mathrm{g} / \mathrm{ml})$ & Catalase $(\mathrm{mU} / \mathrm{mg})$ \\
\hline Control & $1.947 \pm 0.15$ & $99.527 \pm 11$ \\
\hline HFD per se & $6.45 \pm 0.51^{\#}$ & $49.57 \pm 6.8^{\#}$ \\
\hline $\mathrm{HFD}+\mathrm{MCAO}$ & $10.9 \pm 1.42^{\# \# \#}$ & $24.7 \pm 7.5^{\# \# \#}$ \\
\hline $\mathrm{HFD}+\mathrm{GL}(50 \mathrm{mg} / \mathrm{kg})+\mathrm{MCAO}$ & $6.6 \pm 0.87^{*}$ & $56.43 \pm 2.59^{*}$ \\
\hline $\mathrm{HFD}+\mathrm{GL}(100 \mathrm{mg} / \mathrm{kg})+\mathrm{MCAO}$ & $5.88 \pm 0.85^{* *}$ & $67.82 \pm 1.59 * *$ \\
\hline $\mathrm{HFD}+\mathrm{ASP}(100 \mathrm{mg} / \mathrm{kg})+\mathrm{MCAO}$ & $6.25 \pm 0.59 * *$ & $57.39 \pm 4.2 * *$ \\
\hline $\mathrm{HFD}+\mathrm{ATORVA}(20 \mathrm{mg} / \mathrm{kg})+\mathrm{MCAO}$ & $6.35 \pm 0.72 * *$ & $59.68 \pm 2.7 * *$ \\
\hline $\mathrm{HFD}+\mathrm{ASP}(100 \mathrm{mg} / \mathrm{kg})+\mathrm{GL}(50 \mathrm{mg} / \mathrm{kg})+\mathrm{MCAO}$ & $3.9 \pm 0.38 * *$ & $71.2 \pm 8.3^{* *}$ \\
\hline $\mathrm{HFD}+\mathrm{ASP}(100 \mathrm{mg} / \mathrm{kg})+\mathrm{GL}(100 \mathrm{mg} / \mathrm{kg})+\mathrm{MCAO}$ & $3.37 \pm 0.24^{* * *}$ & $78.18 \pm 6.38^{* * *}$ \\
\hline
\end{tabular}

Table 3 Effect of pretreatment of guggulipid, aspirin, atorvastatin and their co-administration on nitrite release and catalase levels in high fat diet fed middle cerebral artery occluded rats.

\section{Effect of pretreatment of guggulipid, aspirin,}

atorvastatin and their co-administration on infarct size in high fat diet fed middle cerebral artery occluded rats The infarction sizes were increased in HFD and HFD + MCAO group as compared with control group $(p<0.01)$. The infarction size (the mean infarction areas) was significantly decreased in pretreated groups of guggulipid, atorvastatin, and guggulipid and aspirin combination groups when compared with HFD and HFD + MCAO ( $<<0.01 ; 0$ Fig. 3,4).

\section{Discussion}

Cerebral ischemia is the second leading cause of death and the third leading role of morbidity in worldwide $[1,30]$. In cerebral ischemia, there is obstruction in blood supply to brain which leads to necrosis in neuronal tissue of brain [31]. The large majority ( $85 \%$ ) of strokes in the western world are ischemic, that is, a stroke resulting from an occlusion of a major cerebral artery, commonly the middle cerebral artery (MCA) by a thrombus or embolism [32]. Cerebral ischemia by reperfusion is an abnormal condition characterized by an initial restriction of blood supply to brain followed by the subsequent restoration of blood flow and concomitant reoxygenation [33].

According to some experimental studies, it was found that either HFD or genetically induced obesity was accompanied with increased cerebrovascular surrounding, produced hypertension and increased infarct size in either transient or permanent focal ischemic model $[4,7]$. In another study it was observed that 8

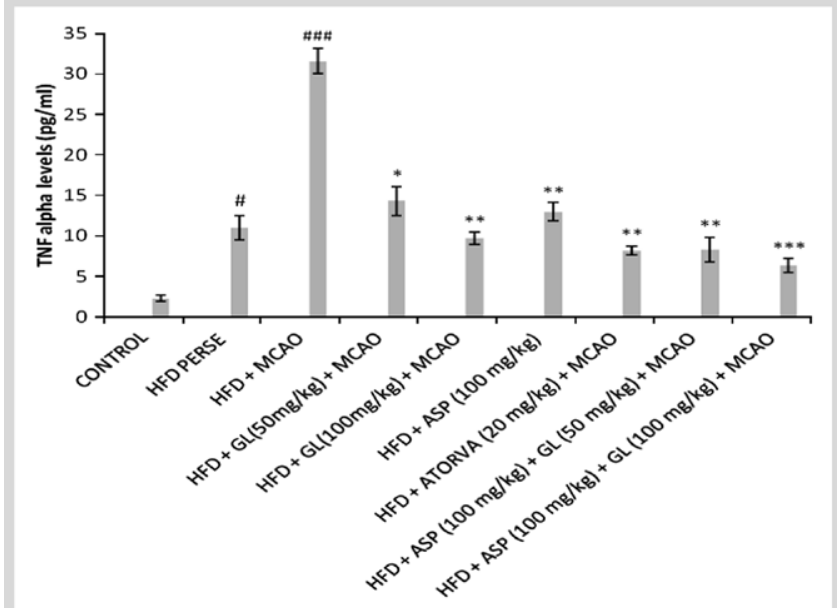

Fig. 2 Effect of pretreatment of guggulipid, aspirin, atorvastatin and their co-administration on TNFa in high fat diet fed middle cerebral artery occluded rats. All the values were expressed as the mean \pm SEM. All group means were compared by one-way analysis of variance (ANOVA) for statistical analysis followed by Dunnett's t-test. $N=$ number of animals 6 , ${ }^{\#} \mathrm{p}<0.05,{ }^{\# \# \#} \mathrm{p}<0.001$ vs. control group; ${ }^{*} \mathrm{p}<0.05,{ }^{* *} \mathrm{p}<0.01{ }^{* * *} \mathrm{p}<$ 0.001 vs. HFD + MCAO. HFD - high fat diet, MCAO - middle cerebral artery occlusion, GL - guggulipid, ASP - aspirin, ATORVA - atorvastatin.

weeks feeding of a high fat diet prior to induction of ischemia altered normal recovery patterns of sensorimotor and cognitive task compared to the standard diet. HFD significantly worsened the 
extent of the neuronal injury and promoted a significantly greater loss of cortical tissue following cortical contusion injuries [8].

Guggulipid has been marketed in India since 1988 as a hypolipidemic agent. This Indian traditional medicine guggulipid though tried and found useful in various disorders like arthritis, inflammation, obesity, cardiac protection, anti-ulcer, anti-epileptic and

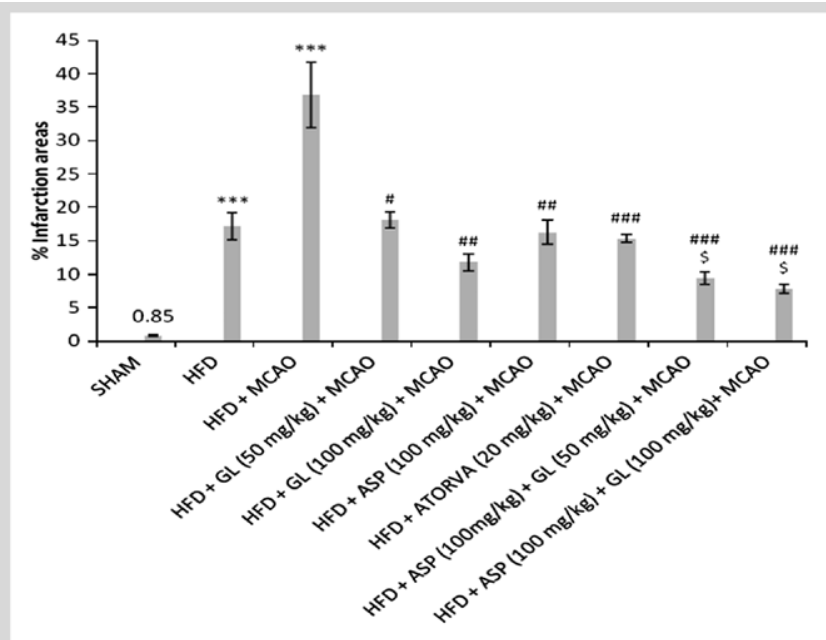

Fig. 3 Effect of pretreatment of guggulipid, aspirin, atorvastatin and their co-administration on infarction size in high fat diet fed middle cerebral artery occluded (MCAO) rats. All the values were expressed as the mean \pm SEM. All group means were compared by one-way analysis of variance (ANOVA) for statistical analysis followed by Dunnett's t-test. $\mathrm{N}=$ number of animals $6,{ }^{*}{ }^{*} \mathrm{p}<0.05$ vs. Sham, ${ }^{\#} \mathrm{p}<0.05,{ }^{\# \#} \mathrm{p}<0.01$, $\# \# \#<0.01$ vs. HFD + MCAO group, ${ }^{\$} \mathrm{p}<0.05$ vs. HFD group. Significance by one-way analysis of variance (ANOVA) followed by Dunnett's't' test. HFD - high fat diet, MCAO - middle cerebral artery occlusion, GL - guggulipid, ASP - aspirin, ATORVA - atorvastatin. disorders of lipid metabolism [1]. Previously it was chemically analyzed and revealed that the compounds responsible for the hypolipidemic activity of gum guggul were the isomers E- and Z-guggulsterone [14]. Guggulipid showed potent memory enhancing and antioxidant activities in the streptozotocin induced mouse model of dementia [34]. Guggulsterones, the active components of guggulipid inhibit NF-kB activation supporting its anti-inflammatory activity [14,35] and effective against myocardial infarction because guggulsterone inhibits platelet aggregation and provide protection against myocardial ischemia induced by isoproterenol [36].

It has been reported that there is a significant impairment of motor performance in middle cerebral occluded rats and high fat diet rat $[1,6,36]$. The possible reason of this dysfunction may be the neuronal damage in the territory of middle cerebral artery. The significant improvement was observed in motor performance of pre-treatment group of guggulipid, atorvastatin, and aspirin when compared with HFD and HFD + MCAO group. The improvement in motor performance may be a sign of reduction in ischemic injury in territory region. Pretreatment of guggulipid and aspirin showed more significant improvement in motor performance of the rats followed by MCAO. Various studies reported the production of free radical production or reactive oxygen species (ROS) in reperfusion induced cerebral ischemia and high fat diet induced obesity which can cause neuronal damage due to oxidation of cellular components like proteins, lipids alteration of signaling pathways that leads to damage of neuronal cells $[2,37]$. High fat diet and middle cerebral artery occlusion significantly enhanced the TBARs levels, which is marker of lipid peroxidation $[38,39]$. Various studies suggested that elevated TBARs levels could be responsible for oxidative stress induced neuronal injury $[40,41]$. In our study TBARs levels were significantly reduced in pretreated groups showing the antioxidant properties which are responsible for reducing oxidative damage.
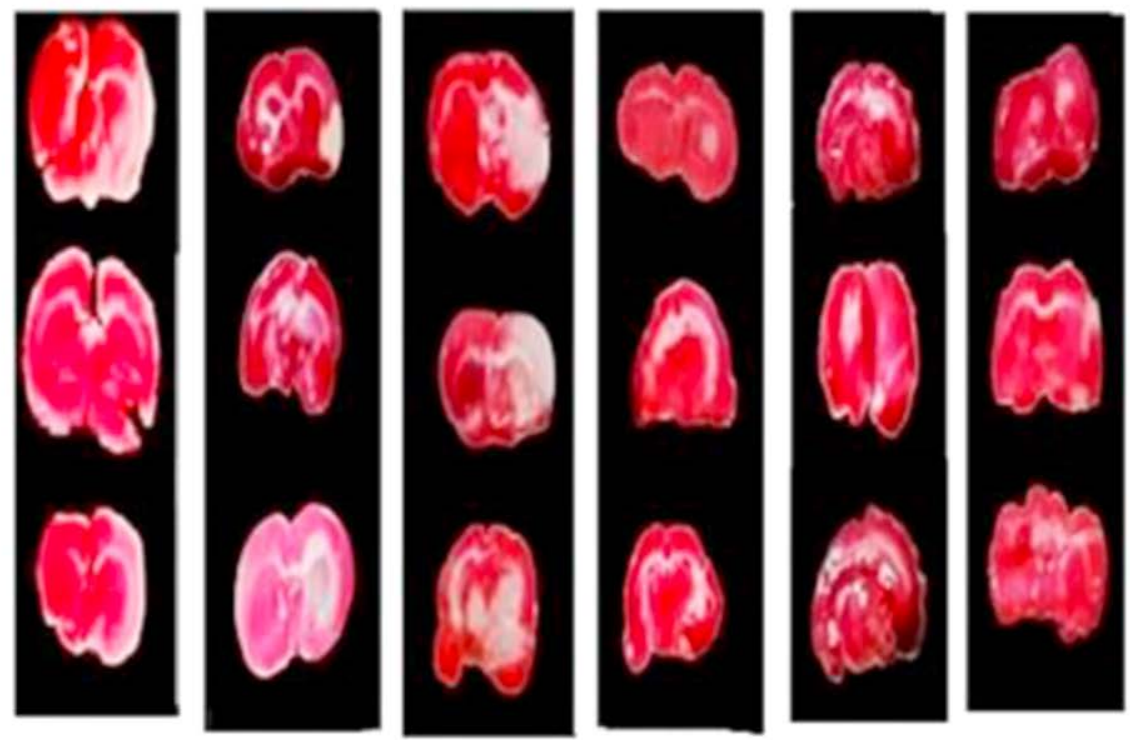

SHLM

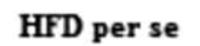

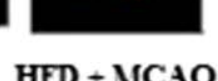

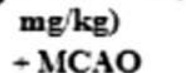

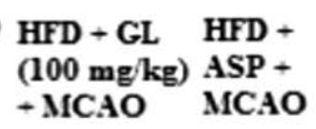

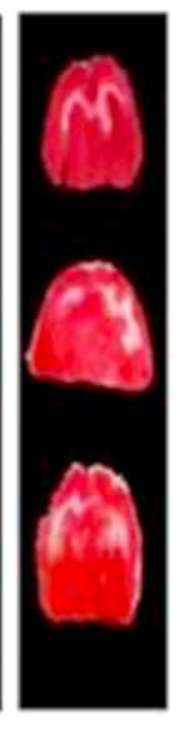

HFD + ATORVA $+\mathrm{MCAO}$
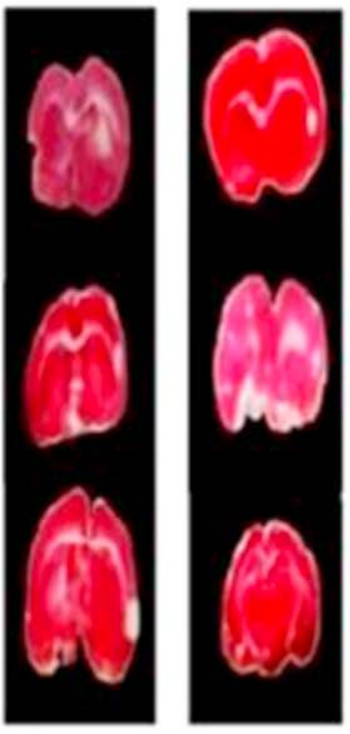

HFD + ASP

+ GL (50 $\mathbf{m g} / \mathbf{k g})+$ MCAO

Fig. 4 Effect of pretreatment of guggulipid, aspirin, atorvastatin and their co-administration on Triphenyl Tetrazolium Chloride (TTC) dye staining of brain slices in high fat diet fed middle cerebral artery occluded (MCAO) rats. HFD - high fat diet, MCAO - middle cerebral artery occlusion, GL - guggulipid, ASP aspirin, ATORVA - atorvastatin. (Color figure available online only). 
Endogenous antioxidants like GSH, SOD and catalase act as detoxifying agents to detoxify the overproduction of free radicals. Physiologically SOD catalyzes the conversion of superoxide anions to hydrogen peroxide and molecular oxygen. Catalase and GSH are involved in scavenging of hydrogen peroxide [4]. In the present study GSH, catalase and SOD levels were significantly reduced in HFD and HFD + MCAO group which could be due to detoxifying the rapidly generated reactive oxygen species caused by high fat diet and acute ischemic reperfusion induced brain injury. Our study also observed a rise in GSH, catalase and SOD levels in pretreatment groups of guggulipid, atorvastatin and aspirin. In combination group of guggulipid and aspirin, GSH, catalase and SOD levels were also more significantly increased as compared with HFD and HFD + MCAO group, indicating that the elevation of these antioxidant enzymes levels were due to the antioxidant properties of pretreated drugs. Our observations are supported with other earlier reported observations $[1,37]$.

There was an elevation of nitric oxide (NO) level found in HFD and HFD + MCAO group of rats. Pretreated drugs significantly reduced the potentiated NO release followed by high fat diet and middle cerebral artery occlusion. It was demonstrated from various studies that high levels of NO production participated for exacerbation of neuronal damage $[18,42]$. Hence the guggulipid pretreatment showed the neuroprotective property.

It was known from various studies that ischemic stroke is evoked by inflammatory reaction $[27,40]$. Brain inflammation produced during reperfusion is due to the accumulation of inflammatory cells and microvascular dysfunction in damaged areas of neuronal tissue [43].

Previous study supported that HFD cause neuro-inflammation which is also responsible for the neuronal injury and or neuronal cells death [44]. Tumor necrosis factor $\alpha\left(\mathrm{TNF}_{\alpha}\right)$ an inflammatory cytokine produced by endothelial cells, astrocytes, macrophages and neuronal cells promote the inflammatory reactions in the brain. In the present study pretreatment of guggulipid, atorvastatin and aspirin showed significant reduction in the inflammatory marker like $\mathrm{TNF}_{\alpha}$. There was a more significant improvement observed in combination group of guggulipid and aspirin. It was also suggested from various study that guggulipid showed antiinflammatory property in neuronal cells $[18,40]$.

Prominent infarction areas were seen in brain sections of HFD and HFD+MCAO groups by TTC dye staining. The infarction areas were significantly reduced in pretreatment groups of guggulipid, atorvastatin, aspirin as compared with HFD and HFD + MCAO groups of rats. In combination group of guggulipid infarctions were less as compared with individual drug of guggulipid, aspirin and atorvastatin. Therefore, the results of the present study demonstrated that chronic pre-treatment of guggulipid may be helpful in reducing the symptoms of cerebral ischemia in rats.

\section{Conclusion}

\section{$\checkmark$}

On the basis of results from this study, it may be concluded that guggulipid reduces the ischemia induced injury, ameliorated the neurobehavioral deficits, and reduced oxidative stress, inflammatory marker and infarct size. With the additional antiischemic activity observed in this study, the previous reported activity of guggulipid such as anti-hyperlipedemic, anti-inflammatory, anti-platelet, antithrombotic and anti-atherosclerotic effects may be useful in reversing the symptoms and may offer significant neuroprotection in cerebral ischemia. Our results are preliminary; more research is warranted to confirm the therapeutic role of guggulipid in cerebral ischemia.

\section{Acknowledgement}

$\nabla$

This research work was carried out and financially supported by the University Grant Commission, New Delhi, India (2013-16) (F. No: 42-689/2013) (SR)

\section{Conflict of Interest}

\section{$\nabla$}

The authors have no conflict of interest to disclose.

\section{References}

1 Ahmad MA, Najmi AK, Mujeeb M et al. Neuroprotective effect of guggulipid alone and in combination with aspirin on middle cerebral artery occlusion (MCAO) model of focal cerebral ischemia in rats. Toxicol Mech Methods 2014; 24: 438-447

2 Bora KS, Sharma A. Neuroprotective effect of Artemisia absinthium L. on focal ischemia and reperfusion-induced cerebral injury. J Ethnopharmacol 2010; 129: 403-409

3 Grau AJ, Weimar C, Buggle F et al. Stroke data bank risk factors, outcome, and treatment in subtypes of ischemic stroke: the German stroke data bank. Stroke 2001; 32: 2559-2566

4 Li W, Prakash R, Chawla D et al. Early effects of high fat diet on neurovascular function and focal ischemic brain injury. Am Physiol Soc 2013; 304: 1001-1008

5 Osmond JM, Mintz JD, Dalton B et al. Obesity increases blood pressure, cerebral vascular remodeling, and severity of stroke in the Zucker rat. Hypertension 2009; 53: 381-386

6 Sarikaya H, Arnold M, Engelter ST et al. Outcome of intravenous thrombolysis in stroke patients weighing over $100 \mathrm{~kg}$. Cerebrovasc Dis 2011; 32: 201-206

7 Deutsch C, Portik-Dobos V, Smith AD et al. Diet-induced obesity causes cerebral vessel remodeling and increases the damage caused by ischemic stroke. Microvascular Research 2009; 78: 100-106

8 Hoane M.R, Swan AA, Heck SE. The effects of a high-fat sucrose diet on functional outcome following cortical contusion injury in the rat. Behavioral Brain Research 2011; 223: 119-124

9 Gupta YK, Briyal S, Gulati A. Therapeutic potential of herbal drugs in cerebral ischemia. Indian J Physiol Pharmacology 2010; 54: 99-122

10 Jonas $S$. Prophylactic pharmacologic neuroprotection against focal cerebral ischemia. Ann NY Acad Science 1995; 765: 21-25

11 MOH Clinical Practice Guidelines: Feb 2009;

12 Hennessy MJ, Britton TC. Transient ischemic attacks: evaluation and management. Int J Clin Pract 2000; 54: 432-436

13 Jauch EC, Saver JL, Adams HP et al. Guidelines for the early management of patients with acute ischemic stroke. Stroke 2013; 44: 870-947

14 Urizar NL, Moore DD. Guggulipd: a natural cholesterol-lowering agent. Ann Rev Nutr 2013; 23: 303-313

15 Deng $R$. Therapeutic effects of guggul and its constituent guggulsterone: cardiovascular benefits. Cardiovasc Drug Rev 2007; 25: 375-390

16 Singh RP, Singh R, Ram P et al. Use of Pushkar-Guggul, an indigenous anti-ischemic combination in the management of ischemic heart disease. Int J Pharmacol 1993; 31: 147-160

17 Gebhard C, Stampfli SF, Gebhard CE et al. Guggulsterone, an antiinflammatory phytosterol, inhibits tissue factor and arterial thrombosis. Basic Res Cardiology 2009; 104: 285-294

18 Niranjan R, Kamat PK, Nath C et al. Evaluation of guggulipid and nimesulide on production of inflammatory mediators and GFAP expression in LPS stimulated rat astrocytoma, cell line (C6). J Ethnopharmacology 2010; 127: 625-630

19 Brobst DE, Ding $X$, Creech KL et al. Guggulsterone activates multiple nuclear receptors and induces CYP3A gene expression through the pregnane X receptor. J Pharmacol Exp Ther 2004; 310: 528-535

20 Longa EZ, Carlson S, Cummins R. Reversible middle cerebral artery occlusion without craniectomy in rats. Stroke 1989; 20: 84-91

21 Danish M, Khanam R, Pillai KK et al. Protective effects of histamine $\mathrm{H}_{3}$-receptor ligands in schizophrenic behaviors in experimental models. Pharmacol Report 2012; 64: 191-204 
22 Morisset S, Sahm UG, Traiffort E et al. Atypical neuroleptics enhance histamine turnover in brain via $5 \mathrm{HT}_{2 \mathrm{~A}}$ receptor blockade. J Pharmacol Exp Ther 1999; 288: 590-596

23 Ali A, Ahmed FJ, Pillai KK et al. Evidence of antiepileptic potential of amiloride with neuropharamacological benefits in rodents models of epilepsy and behavior. Epilepsy Behav 2004; 5: 322-328

24 Okhawa $\mathrm{H}$, Ohishi $N$, Yagi K. Assay of lipid peroxides in animals tissue by thiobarbituraic acid reaction. Anal Biochem 1979; 95: 351-358

25 Ellman GL. Tissue sulphydryl groups. Arch Biochem Biophys 1957; 82: 70-73

26 Beauchamp C, Fridovich I. Superoxide dismutase: improved assays and an assay applicable to acrylamide gels. Anal Biochem 1971; 44: 276-287

27 Claiborne A, Greenworld RA. Handbook of method of oxygen free radical research. Boca Raton (FL): CRC Press; 1985: 4: 283-284

28 Aggarwal A, Gaur V, Kumar A. Nitric oxide mechanism in the protective effect of naringin against post-stroke depression (PSD) in mice. Life Science 2010; 86: 928-935

29 Saraf MK, Prabhakar S, Anand A. Neuroprotective effect of Bacopa monniera on ischemia induced brain injury. Pharmacol Biochem Behav 2010; 97: 192-197

30 Hankey GJ. The global and regional burden of stroke. Lancet 2013; 1: 239-240

31 Park SJ, Nam KW, Lee HJ et al. Neuroprotective effects of an alkaloidfree ethyl acetate extract from the root of Sophora flavescens Ait against focal cerebral ischemia in rats. Phytomedicine 2009; 16: 1042-1051

32 Green AR. Pharmacological approaches to acute ischaemic stroke: reperfusion certainly, neuroprotection possibly. Br J Pharmacol 2008; 153: $325-338$

33 Chen C, Venketasubramanian N, Gan RN et al. Danqi Piantang Jiaonang (DJ), a traditional Chinese medicine in post stroke recovery. Stroke 2009; 40: 859-863
34 Saxena G, Singh SP, Pal R. Guggulipd, an extract of Commiphora whighitii with lipid-lowering properties, has protective effects against streptozotocin-induced memory deficits in mice. Pharmacol Biochem Behav 2007; 86: 797-805

$35 \mathrm{Wu} P F$, Zhang $Z$, Wang $F$ et al. Natural compounds from traditional medicinal herbs in the treatment of cerebral ischemia/reperfusion injury. Acta Pharmacol Sin 2010; 31: 1523-1531

36 Ojha SK, Nandave M, Arora S et al. Effect of Commiphora mukul extract on cardiac dysfunction and ventricular function in isoproterenol induced myocardial infarction. Ind J Exp Biology 2008; 46: 646-652

37 Akhtar M, Imam SS, Ahmad MA et al. Neuroprotective study of Nigella sativa-loaded oral provesicular lipid formulation: in vitro and ex vivo study. Drug Delivery 2014; 21: 487-494

$38 \mathrm{Du} \mathrm{J}$, Mei J. 24 Oxidative stress and endothelial dysfunction in high-fat diet-induced obesity in mice. Heart 2011; 97: 8-24

$39 \mathrm{Wu} H T$, He XJ, Hong YK et al. Chemical characterization of lycium barbarum polysaccharides and its inhibition against liver oxidative injury of high-fat mice. Int J Biol Macro 2010; 46: 540-543

40 Gupta YK, Sinha K, Chaoudhary G. Transient focal ischemia induces motor deficit but does not impair the cognitive function in middle cerebral artery occlusion model of stroke in rats. J Neurol Sci 2002; 203: 267-271

41 Sinha K, Degaonkar N, Jagannathan NR et al. Effect of melatonin on ischemia reperfusion injury induced by middle cerebral artery occlusion in rats. Eur J Pharmacol 2001; 428: 185-192

42 Dohare $P$, Varma S, Ray $M$. Curcuma oil modulates the nitric oxide system response to cerebral ischemia/reperfusion injury. Nitric Oxide 2008; 19: 1-11

43 Ding Y, Clark JC. Cerebrovascular injury in stroke. Neurol Res 2006; 28: $3-10$

44 Pratibha VN, Lisa MJ, Lance MB. Momordika charantia attenuates high fat diet associated oxidative stress and neuroinflammation. J Neuroinflammation 2011; 8: 64 doi:10.1186/1742-2094-8-64 